\title{
Lenvatinib Plus Pembrolizumab Improves Outcomes in Previously Untreated Advanced Clear Cell Renal Cell Carcinoma
}

\begin{abstract}
Motzer R, Alekseev B, Rha SY, et al. Lenvatinib plus pembrolizumab or everolimus for advanced renal cell carcinoma [published online ahead of print, 2021 Feb 13]. N Engl J Med. 2021;10.1056/ NEJMoa2035716. doi:10.1056/NEJMoa2035716
\end{abstract}

\section{Study Overview}

Objective. To evaluate the efficacy and safety of lenvatinib in combination with everolimus or pembrolizumab compared with sunitinib alone for the treatment of newly diagnosed advanced clear cell renal cell carcinoma (ccRCC).

Design. Global, multicenter, randomized, open-label, phase 3 trial.

Intervention. Patients were randomized in a 1:1:1 ratio to receive treatment with 1 of 3 regimens: lenvatinib 20 $\mathrm{mg}$ daily plus pembrolizumab $200 \mathrm{mg}$ on day 1 of each 21-day cycle; lenvatinib $18 \mathrm{mg}$ daily plus everolimus $5 \mathrm{mg}$ once daily for each 21-day cycle; or sunitinib $50 \mathrm{mg}$ daily for 4 weeks followed by 2 weeks off. Patients were stratified according to geographic region and Memorial Sloan Kettering Cancer Center (MSKCC) prognostic risk group.

Setting and participants. A total of 1417 patients were screened, and 1069 patients underwent randomization between October 2016 and July 2019: 355 patients were randomized to the lenvatinib plus pembrolizumab group,
357 were randomized to the lenvatinib plus everolimus group, and 357 were randomized to the sunitinib alone group. The patients must have had a diagnosis of previously untreated advanced renal cell carcinoma with a clear-cell component. All the patients need to have a Karnofsky performance status of at least 70 , adequate renal function, and controlled blood pressure with or without antihypertensive medications.

Main outcome measures. The primary endpoint assessed the progression-free survival (PFS) as evaluated by independent review committee using RECIST, version 1.1. Imaging was performed at the time of screening and every 8 weeks thereafter. Secondary endpoints were safety, overall survival (OS), and objective response rate as well as investigator-assessed PFS. Also, they assessed the duration of response. During the treatment period, the safety and adverse events were assessed up to 30 days from the last dose of the trial drug.

Main results. The baseline characteristics were well balanced between the treatment groups. More than $70 \%$

\section{Outcomes Research in Review SECTION EDITORS}

KatRINA F. MATEO, PHD, MPH

CUNY School of Public Health New York, NY
DANIEL ISAAC, DO, MS

Michigan State University East Lansing, Ml
FRED Ko, MD, MS

Icahn School of Medicine at Mount Sinai New York, NY
TAISHI HIRAI, MD

University of Missouri

Columbia, $\mathrm{MO}$
William HUNG, MD, MPH

Icahn School of Medicine

at Mount Sinai

New York, NY 
of enrolled participants were male. Approximately 60\% of participants were MSKCC intermediate risk, $27 \%$ were favorable risk, and 9\% were poor risk. Patients with a PD-L1 combined positive score of $1 \%$ or more represented $30 \%$ of the population. The remainder had a PD-L1 combined positive score of $<1 \%$ (30\%) or such data were not available (38\%). Liver metastases were present in $17 \%$ of patients at baseline in each group, and $70 \%$ of patients had a prior nephrectomy. The data cutoff occurred in August 2020 for PFS and the median follow-up for OS was 26.6 months. Around $40 \%$ of the participants in the lenvatinib plus pembrolizumab group, $18.8 \%$ in the sunitinib group, and $31 \%$ in the lenvatinib plus everolimus group were still receiving trial treatment at data cutoff. The leading cause for discontinuing therapy was disease progression. Approximately 50\% of patients in the lenvatinib/everolimus group and sunitinib group received subsequent checkpoint inhibitor therapy after progression.

The median PFS in the lenvatinib plus pembrolizumab group was significantly longer than in the sunitinib group, 23.9 months vs 9.2 months (hazard ratio [HR], 0.39; 95\% Cl, 0.32-0.49; $P<0.001$ ). The median PFS was also significantly longer in the lenvatinib plus everolimus group compared with sunitinib, 14.7 vs 9.2 months (HR 0.65; 95\% Cl 0.53-0.80; $P<0.001$ ). The PFS benefit favored the lenvatinib combination groups over sunitinib in all subgroups, including the MSKCC prognostic risk groups. The median OS was not reached with any treatment, with $79 \%$ of patients in the lenvatinib plus pembrolizumab group, $66 \%$ of patients in the lenvatinib plus everolimus group, and $70 \%$ in the sunitinib group still alive at 24 months. Survival was significantly longer in the lenvatinib plus pembrolizumab group compared with sunitinib $(\mathrm{HR}, 0.66 ; 95 \% \mathrm{Cl}$, 0.49-0.88; $P=0.005$ ). The OS favored lenvatinib/pembrolizumab over sunitinib in the PD-L1 positive or negative groups. The median duration of response in the lenvatinib plus pembrolizumab group was 25.8 months compared to 16.6 months and 14.6 months in the lenvatinib plus everolimus and sunitinib groups, respectively. Complete response rates were higher in the lenvatinib plus pembrolizumab group (16\%) compared with lenvatinib/everolimus $(9.8 \%)$ or sunitinib $(4.2 \%)$. The median time to response was around 1.9 months in all 3 groups.
The most frequent adverse events seen in all groups were diarrhea, hypertension, fatigue, and nausea. Hypothyroidism was seen more frequently in the lenvatinib plus pembrolizumab group (47\%). Grade 3 adverse events were seen in approximately $80 \%$ of patients in all groups. The most common grade 3 or higher adverse event was hypertension in all 3 groups. The median time for discontinuing treatment due to side effects was 8.97 months in the lenvatinib plus pembrolizumab arm, 5.49 months in the lenvatinib plus everolimus group, and 4.57 months in the sunitinib group. In the lenvatinib plus pembrolizumab group, 15 patients had grade 5 adverse events; 11 participants had fatal events not related to disease progression. In the lenvatinib plus everolimus group, there were 22 patients with grade 5 events, with 10 fatal events not related to disease progression. In the sunitinib group, 11 patients had grade 5 events, and only 2 fatal events were not linked to disease progression.

Conclusion. The combination of lenvatinib plus pembrolizumab significantly prolongs PFS and OS compared with sunitinib in patients with previously untreated and advanced ccRCC. The median OS has not yet been reached.

\section{Commentary}

The results of the current phase 3 CLEAR trial highlight the efficacy and safety of lenvatinib plus pembrolizumab as a first-line treatment in advanced ccRCC. This trial adds to the rapidly growing body of literature supporting the notion that the combination of anti-PD-1 based therapy with either CTLA-4 antibodies or VEGF receptor tyrosine kinase inhibitors (TKI) improves outcomes in previously untreated patients with advanced ccRCC. Previously presented data from Keynote-426 (pembrolizumab plus axitinib), Checkmate-214 (nivolumab plus ipilimumab), and Javelin Renal 101 (Avelumab plus axitinib) have also shown improved outcomes with combination therapy in the frontline setting. ${ }^{1-4}$ While the landscape of therapeutic options in the frontline setting continues to grow, there remains lack of clarity as to how to tailor our therapeutic decisions for specific patient populations. The exception would be nivolumab and ipilimumab, which are currently indicated for IMDC intermediate- or poor-risk patients. 


\section{Outcomes Research in Review}

The combination of VEGFR TKI therapy and PD-1 antibodies provides rapid disease control, with a median time to response in the current study of 1.9 months, and, generally speaking, a low risk of progression in the first 6 months of therapy. While cross-trial comparisons are always problematic, the PFS reported in this study and others with VEGFR TKI and PD-1 antibody combinations is quite impressive and surpasses that noted in Checkmate 214. ${ }^{3}$ While the median OS survival has not yet been reached, the long duration of PFS and complete response rate of $16 \%$ in this study certainly make this an attractive frontline option for newly diagnosed patients with advanced ccRCC. Longer follow-up is needed to confirm the survival benefit noted.

\section{Applications for Clinical Practice}

The current data support the use VEGFR TKI and anti-PD1 therapy in the frontline setting. How to choose between such combination regimens or combination immunotherapy remains unclear, and further biomarker-based assessments are needed to help guide therapeutic decisions for our patients.

-Saud Alsubait, MD

\section{References}

1. Motzer, R, Alekseev B, Rha SY, et al. Lenvatinib plus pembrolizumab or everolimus for advanced renal cell carcinoma [published online ahead of print, 2021 Feb 13]. N Engl J Med. 2021;10.1056/ NEJMoa2035716. doi:10.1056/NEJMoa2035716

2. Rini, BI, Plimack ER, Stus V, et al. Pembrolizumab plus axitinib versus sunitinib for advanced renal-cell carcinoma. N Engl J Med. 2019;380(12):1116-1127.

3. Motzer, RJ, Tannir NM, McDermott DF, et al. Nivolumab plus ipilimumab versus sunitinib in advanced renal-cell carcinoma. $N$ Engl J Med. 2018;378(14):1277-1290.

4. Motzer, RJ, Penkov K, Haanen J, et al. Avelumab plus axitinib versus sunitinib for advanced renal-cell carcinoma. $N$ Engl J Med. 2019;380(12):1103-1115.

\section{CALL FOR MANUSCRIPTS}

- Allergy and immunology

- Cardiovascular disease

- Diabetes mellitus

- Geriatrics and gerontology

- Gl disorders

- Hematology/ oncology

- HIV/AIDS

- Kidney disease

- Mental health

- Pain management

- Population health

- PTSD

- Pulmonary disease

- Rheumatology

- Stroke

- Substance abuse

www.mdedge.com/jcomjournal/page/information-authors. Email your manuscript or queries to eseger@mdedge.com. 